\title{
CROSSED PRODUCTS AND MF ALGEBRAS
}

\author{
WEIHUA Li AND SteFAnOS ORFANOS
}

Abstract. We prove that the crossed product $\mathscr{A} \rtimes_{\alpha} G$ of a unital finitely generated MF algebra $\mathscr{A}$ by a discrete finitely generated amenable residually finite group $G$ is an MF algebra, provided that the action $\alpha$ is almost periodic. This generalizes a result of Hadwin and Shen. We also construct two examples of crossed product $C^{*}$-algebras whose BDF Ext semigroups are not groups.

Mathematics subject classification (2010): 46L05.

Keywords and phrases: MF algebras, crossed products, BDF Ext semigroups, amenable groups, residually finite groups.

\section{REFERENCES}

[1] B. BlackAdar And E. Kirchberg, Generalized inductive limits of finite-dimensional $C^{*}$ algebras, Math. Ann. 307 (3) (1997) 343-380.

[2] L. Brown, R. Douglas And P. Fillmore, Extensions of $C^{*}$-algebras and $K$-homology, Ann. Math. 105 (1977) 265-324.

[3] U. HaAgerup And S. ThorbjøRnsen, A new application of random matrices: $\operatorname{Ext}\left(C_{r e d}^{*}\left(F_{2}\right)\right)$ is not a group, Ann. Math. (2) 162 (2) (2005) 711-775.

[4] D. Hadwin And J. SHEn, Some examples of Blackadar and Kirchberg's MF algebras, Internat. J. Math. 21 (10) (2010) 1239-1266.

[5] S. ORfanos, Quasidiagonality of crossed products, J. Operator Theory 66 (1) (2011) 209-216.

[6] M. Pimsner AND D. Voiculescu, Exact sequences for $K$-groups and Ext-groups of certain crossed-product $C^{*}$-algebras, J. Operator Theory 4 (1) (1980) 93-118.

[7] J. Rosenberg, Quasidiagonality and nuclearity (appendix to Strongly quasidiagonal operators by D. Hadwin), J. Operator Theory 18 (1987) 15-18.

[8] D. Voiculescu, The topological version of free entropy, Lett. Math. Phys. 62 (1) (2002) 71-82. 